\title{
Bounded Speed of Propagation for Solutions to Radiative Transfer Equations
}

\author{
Benoît Perthame $^{1 \star}$ and Juan Luis Vazquez ${ }^{2 \star \star}$ \\ ${ }^{1}$ Département de Mathématiques, Université d'Orléans, B.P. 6759, F-45067 Orleans Cedex 02, France \\ 2 Departamento de Matemáticas, Universidad Autónoma de Madrid, Cantoblanco, E-28049 Madrid, \\ Spain
}

Abstract. The Radiative Transfer Equation is the nonlinear transport equation

$$
\partial_{t} f+\frac{1}{\varepsilon} v \nabla_{x} f+\frac{1}{\varepsilon^{2}} \sigma(\tilde{f})(f-\tilde{f})=0,
$$

where $\tilde{f}(x, t)=f f(x, v, t) d v$ denotes the average of $f(x, ., t)$ on the unit sphere: $|v|=1$. It describes the absorption and emission of photons in a hot medium. As the mean free path $\varepsilon$ goes to $0, f_{\varepsilon}$ converges to a solution of the Porous Medium Equation $\partial_{t} u=\Delta F(u)$, with $F^{\prime}(u)=(N \sigma(u))^{-1}$. Since $\sigma$ blows up at $u=0$, solutions to the PME propagate with finite speed. Specifically if $u(\cdot, 0)$ has compact support in $\mathbf{R}^{N}$ so does $u(\cdot, t)$ for every $t>0$ and the sets $\Omega(t)=\{x: u(x, t)>0\}_{t>0}$ form an expanding family as $t$ increases, and $U \boldsymbol{\Omega}(t)=\mathbf{R}^{n}$. We show in this paper that these propagation properties hold for the solutions $f_{\varepsilon}$ of the RTE for all small $\varepsilon$. Moreover, the growth of the support of $f_{\varepsilon}$ is uniform in $\varepsilon$. Our proofs rely on the construction of explicit solutions (of the travelling wave type) and subsolutions to the RTE. To our knowledge, this is the first example of a kinetic equation with high velocities where localized data propagate always with bounded speed. For Vlasov-Poisson equations, this arises only for particular initial data.

\section{Introduction}

In this paper we show that the property of finite propagation of disturbances from 0 , well known for the solutions of the Porous Medium Equation: $u_{t}=\Delta u^{m}, m>1$, holds also for the Radiative Transfer Equation. In order to be specific, we consider

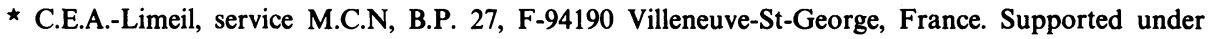
contract with E.N.S., $n^{\circ} 1992-44$

$\star \star$ Supported by E.E.C. contact $n^{\circ}$ SC1-0019-C and DGICYT(Spain)-Project $n^{\circ}$ PB86-0112-CO200
} 
nonnegative solutions $f_{\varepsilon}=f_{\varepsilon}(x, v, t)$ to the Cauchy problem

$$
\left\{\begin{array}{lll}
\partial_{t} f+\frac{1}{\varepsilon} v \cdot \nabla_{x} f+\frac{1}{\varepsilon^{2}} \sigma(\tilde{f})(f-\tilde{f})=0 & \text { for } & x \in \mathbf{R}^{N}, \quad v \in S^{N-1}, \quad t \geqq 0, \\
f(x, v, 0)=f_{0}(x, v) & \text { for } \quad x \in \mathbf{R}^{N}, \quad v \in S^{N-1},
\end{array}\right.
$$

where $S^{N-1}$ is the unit sphere in $\mathbf{R}^{N}, \tilde{f}$ represents the average of $f$ in the velocity variable

$$
\tilde{f}(x, t)=\left|S^{N-1}\right|^{-1} \int_{S^{N-1}} f(x, v, t) d v
$$

and the initial function $f_{0}$ satisfies: $f_{0} \in L^{1}\left(\mathbf{R}^{N} \times S^{N-1}\right), f_{0} \geqq 0$ a.e. and has compact support in the $x$ variable.

Since the transport operator governs this equation, the maximal speed of propagation in $(0.1)_{\varepsilon}$ is $1 / \varepsilon$ and the diameter of the support grows at most like $D_{0}+t / \varepsilon$. We are going to prove that these intuitive bounds are not realistic for small $\varepsilon$ in the physically relevant case where the opacity function $\sigma$ blows up at 0 . We show that the support indeed expands but its diameter grows at most like $D_{0}+C t^{1 / 2}$ with constants independent of $\varepsilon$.

This somewhat surprising consequence of the presence of the nonlinear term $\varepsilon^{-2} \sigma(\tilde{f})(\tilde{f}-f)$ can be explained by the behaviour of $(0.1)_{\varepsilon}$ as $\varepsilon \rightarrow 0$. It is then known, cf. $[2,3]$, that $f_{\varepsilon}(x, v, t)$ converges to a solution $u(x, t)$ of the generalized porous medium equation

$$
u_{t}=\Delta F(u), \quad t>0, \quad x \in \mathbf{R}^{N},
$$

where $F$ is given by $F(0)=0$ and

$$
F^{\prime}(u)=1 /(N \sigma(u))>0 \text { if } u>0 .
$$

The support of a solution to (0.3) spreads out with a finite speed if and only if $F^{\prime}$ degenerates at 0 in the following sense: the integral $\int\left(F^{\prime}(u) / u\right) d u$ converges at $u=0$, cf. $[10,7,11,4]$.

Our proofs will rely on the analogy between $(0.1)_{\varepsilon}$ and (0.3). In particular, we construct in Sect. 1 an explicit travelling wave solution to $(0.1)_{\varepsilon}$ inspired by known solutions of (0.3), and use comparison arguments (Sect. 2) to establish bounds on the growth of the support. Construction of a suitable subsolution allows to show that the support does not shrink in $x$; in fact it expands in time to cover the whole space $\mathbf{R}^{n}$ (Sect. 3). It is worth remarking that the support actually expands in $v$ to the whole sphere $S^{N-1}$ instantaneously at all points where the $v$-average is positive at $t=0$.

The precise hypotheses imposed on the opacity $\sigma$ are the following: Firstly, $\sigma \in C\left(\mathbf{R}^{+}, \mathbf{R}^{+}\right)$with a possible blow up at 0 and

$$
\begin{gathered}
\sigma(u) \geqq \sigma_{R}>0 \quad \text { for } \quad 0<u<R, \\
\int_{0}^{1} \frac{d u}{u \sigma(u)}<\infty, \quad \int_{1}^{\infty} \frac{d u}{u \sigma(u)}=\infty .
\end{gathered}
$$


Hypothesis (H1) is used in $[2,6]$. As for $(\mathrm{H} 2)$, the convergence of the integral at 0 allows to define the pressure function in the next chapter and is essential for finite propagation. The divergence at $\infty$ is not so important. In order to get a comparison principle between sub- and supersolutions to $(0.1)_{e}$, we have to add the assumptions:

$$
\sigma(u) \text { is non-increasing, } \sigma(u) u \text { is non-decreasing, }
$$

which imply the accretivity of the differential operator, see [9] or Appendix. Observe that the first condition means that $F$ is convex, while the second condition implies that $F(u) \geqq c u^{2}$ for small $u>0$.

We denote by $\left|S^{N-1}\right|=2 \pi^{N / 2} / \Gamma(N / 2)$ the usual $(N-1)$-measure of the unit sphere and denote by $\nabla, \Delta$ the spatial operators $\nabla_{X}, \Delta_{X}$. The main properties of the porous medium equation can be found in the survey paper [1].

\section{Travelling Waves}

This section is devoted to the construction of exact travelling waves for the Radiative Transfer Equation (0.1). Since we do not make use of comparison principles, the accretivity assumptions $(\mathrm{H} 3)$ are not necessary.

By a travelling-wave, we mean a wave which travels with velocity $c$ in $(x, t)$-space, with fixed profile in $v$. We consider such functions of the form

$$
f(x, v, t)=u\left(x \cdot c+|c|^{2} t\right) \cdot g(v) .
$$

Our solution will be so constructed that $g(v) \cong 1$ when $\varepsilon$ is close to 0 . In this way, the dependence on $v$ is lost in the limit $\varepsilon \rightarrow 0$ and we recover a travelling-wave solution to the P.M.E. (0.3).

With this motivation the construction proceeds as follows. Inspired by the P.M.E. theory, cf. [8], we define the pressure associated with $u$ by

$$
P(u)=\int_{0}^{u} \frac{1}{t \sigma(t)} d t, \quad u \geqq 0
$$

and let $P_{\lambda}(u)=P(u) / \lambda$. Thanks to $(\mathrm{H} 2), P_{\lambda}$ is well defined, increasing, continuous and $P(0)=0, P(\infty)=\infty$. Therefore, $P^{-1}$ is a well defined, continuous, increasing function $\mathbf{R}^{+} \rightarrow \mathbf{R}^{+}$with $P_{\lambda}^{-1}(\infty)=\infty, P_{\lambda}^{-1}(0)=0$.

Let now $u_{\lambda}$ be defined as

$$
u_{\lambda}(s)=P_{\lambda}^{-1}(s) \text { for } s \geqq 0, u_{\lambda}(s)=0 \text { for } s \leqq 0 .
$$

Then $u_{\lambda}^{\prime}=\lambda u_{\lambda} \sigma\left(u_{\lambda}\right)$. It follows that the function

$$
u_{\lambda, c}(x, t)=u_{\lambda}\left(x \cdot c+|c|^{2} t\right)
$$

is indeed a travelling-wave solution to

$$
\partial_{t} u-\Delta F_{\lambda}(u)=0,
$$

with

$$
F_{\lambda}^{\prime}(u)=1 /(\lambda \sigma(u))
$$


since $\left(F_{\lambda}\left(u_{\lambda}(s)\right)\right)^{\prime}=u_{\lambda}(s)$. Next, we select a particular ad hoc form of $g$ to obtain the following candidate to be a solution of $(0.1)_{\varepsilon}$ :

$$
f_{\varepsilon}(x, v, t)=\frac{u_{\lambda}\left(x \cdot c+|c|^{2} t\right)}{1+\lambda \varepsilon c \cdot v+\lambda \varepsilon^{2}|c|^{2}}
$$

We have

Theorem 1. Under assumptions (H1), (H2), for every $\varepsilon>0$ and $c \in \mathbf{R}^{N}$ with $0<\varepsilon|c|<1$ if $N \leqq 3,0<\varepsilon|c|<1 /(N-2)$ otherwise, there exists a unique $\lambda=\lambda(\varepsilon|c|)>0$ such that the function $f_{\varepsilon}$ given by (1.7) is an exact nonnegative solution to $(0.1)_{\varepsilon}$.

Remark 1. As $\varepsilon$ goes to 0 with fixed $c \neq 0, \lambda(\varepsilon|c|)$ goes to $\lambda(0)=N$ and we recover the function $u_{N, c}$ in (1.4), solution to (0.3).

Remark 2. For a given $\varepsilon>0$, the limitation $c<1 / \varepsilon$ is essential since the speed of propagation for Eq. $(0.1)$ is clearly equal or less than $1 / \varepsilon$. The restriction on $c$ for $N>3$ is technical.

The proof of this Theorem relies on a calculation given in

Lemma 2. For every $\alpha>0$ with $\alpha<1$ if $N \leqq 3, \alpha<1 /(N-2)$ otherwise, there exists a unique $\lambda(\alpha)>0$ such that

$$
g=g_{\lambda, \alpha}(v)=\frac{1}{1+\lambda \alpha v_{1}+\lambda \alpha^{2}}
$$

with $v=\left(v_{1}, \ldots, v_{N}\right)$, is positive and satisfies

$$
\underset{S^{N-1}}{f} g(v) d v=1
$$

Moreover $\lambda(\alpha) \rightarrow N$ as $\alpha \rightarrow 0$.

Proof. Let us consider only values of $\lambda$ in the interval $[0,1 / \alpha(1-\alpha))$ so that $g(v)$ remains positive. Then, for a given $\alpha$, we define

$$
\varphi(\lambda)=\operatorname{s}_{s^{N-1}} \frac{d v}{1+\lambda \alpha v_{1}+\lambda \alpha^{2}} .
$$

One easily checks that, for $0 \leqq \lambda \leqq 1 / \alpha(1-\alpha)=\lambda_{1}, \varphi(\lambda)$ is a convex function with $\varphi(0)=1, \varphi^{\prime}(0)=-\alpha^{2}<0$. Therefore, Lemma 2 follows if we can show that $\varphi\left(\lambda_{1}\right)>1$. We have

$$
\varphi\left(\lambda_{1}\right)=(1-\alpha) f \frac{d v}{1+v_{1}}=\frac{1-\alpha}{\left|S^{N-1}\right|} \int_{S^{N-1}} \frac{d v}{1-v_{1}^{2}}=2(1-\alpha) \int_{0}^{1}\left(1-x^{2}\right)^{(N-5) / 2} d x \frac{\left|S^{N-2}\right|}{\left|S^{N-1}\right|} .
$$

Therefore, $\varphi\left(\lambda_{1}\right)=\infty$ for $N=1,2,3$ and

$$
\varphi\left(\lambda_{1}\right)=(1-\alpha) \frac{N-2}{N-3} \text { if } N>3 .
$$

Thus, with the restriction $\alpha<1 /(N-2)$ the conclusion (1.9) holds for a unique $\lambda=\lambda(\alpha)$. 
Moreover, for fixed $\lambda>0$ and small $\alpha$, we have

$$
\varphi(\lambda)=1-\lambda \alpha^{2}+\frac{\lambda^{2} \alpha^{2}}{N}+O\left(\alpha^{3}\right) .
$$

Hence, if $\lambda>N, \varphi(\lambda)>1$ for $\alpha$ small enough and if $\lambda<N, \varphi(\lambda)<1$ also for $\alpha \cong 0$, consequently $\lambda(\alpha) \rightarrow N$ as $\alpha$ goes to $0_{\# \text {. }}$

Proof of Theorem 1. Let $\varepsilon>0$ and $c \in \mathbf{R}^{N}$ satisfy the assumption above. We may always assume that $c$ has the direction of the $x_{1}$-axis. We then take $f_{\varepsilon}$ as in (1.7), i.e. $f_{\varepsilon}=u_{\lambda, c} \cdot g_{\lambda, \varepsilon|c|}$, with $\lambda=\lambda(\varepsilon|c|)$ as in Lemma 2. Then $\tilde{f}_{\varepsilon}=u_{\lambda, c}$ and

$$
\begin{aligned}
\varepsilon^{2} \partial_{t} f_{\varepsilon}+\varepsilon v \cdot \nabla f_{\varepsilon}+\sigma\left(\tilde{f}_{\varepsilon}\right)\left(f_{\varepsilon}-\tilde{f}_{\varepsilon}\right)= & g(v) u_{\lambda}^{\prime}(\xi)\left[\varepsilon^{2}|c|^{2}+\varepsilon v \cdot c\right] \\
& +\sigma\left(u_{\lambda}(\xi)\right) u_{\lambda}(\xi)(g(v)-1)=\left({ }^{*}\right),
\end{aligned}
$$

where $\xi=x \cdot c+|c|^{2} t$. Using $u_{\lambda}=\lambda u_{\lambda} \sigma\left(u_{\lambda}\right)$, we get

$$
\left(^{*}\right)=\sigma\left(u_{\lambda}(\xi)\right) u_{\lambda}(\xi)\left[g(v)\left(1+\lambda \varepsilon v \cdot c+\lambda \varepsilon^{2}|c|^{2}\right)-1\right]=0 .
$$

This computation holds for $\xi \neq 0$, but since $f_{\varepsilon}$ is continuous, Eq. $(0.1)_{\varepsilon}$ holds in $\mathscr{D}^{\prime}\left(\mathbf{R}^{2 N} \times \mathbf{R}\right)$ and Theorem 1 is proved $\#$.

\section{Finite Speed of Propagation}

We now use the travelling waves constructed above to establish the finite speed of propagation of the support for bounded solutions of $(0.1)_{\varepsilon}$. Since we will make use of a comparison argument, we need assumptions $(\mathrm{H} 1),(\mathrm{H} 2),(\mathrm{H} 3)$ on $\sigma$. We also assume that $0<\varepsilon<1$.

Theorem 3. Let (H1)-(H3) hold and let $f_{0}(x, v)$ satisfy: $0 \leqq f_{0} \leqq M, \operatorname{supp}\left(f_{0}\right) \subset B_{R}$ for some $M, R>0$. If $f_{\varepsilon}$ is the solution of $(0.1)_{\varepsilon}$, then

$$
\operatorname{supp} f_{\varepsilon}(\cdot, v, t) \subset B_{R(t)} \text {, }
$$

where

$$
R(t) \leqq R+(A t)^{1 / 2}
$$

with $A \leqq c_{N} \cdot P_{N}\left(c_{N} M\right), c_{N}>0$. Moreover, for $\delta=P_{N}(M) \varepsilon^{2} / t$ close to 0 , we have $A=2 P_{N}\left(M+M \delta^{1 / 2}\right)+\rho(\delta)$, where $\rho \rightarrow 0$ as $\delta \rightarrow 0$.

Remark. In particular, letting $\varepsilon \rightarrow 0$, we recover for the Porous Medium Equation the bound (cf. [8])

$$
R(t) \leqq R+2\left(P_{N}(M) t\right)^{1 / 2} .
$$

The rate $t^{1 / 2}$ is optimal for $L^{\infty}$ initial data, since it is the precise rate of expansion of the support for selfsimilar solutions of the form $u(x, t)=\varphi\left(x t^{-1 / 2}\right)$ constructed e.g. in [5]. See [12] for a detailed investigation of growth rates.

Proof. Let $\varepsilon>0$ and choose $c \neq 0$ such that

$$
\varepsilon|c|<1 \text { if } N \leqq 3, \quad \varepsilon|c|<1 /(N-2) \text { otherwise. }
$$


We also choose $t_{0}>0$ such that

$$
|c|^{2} t_{0}-R|c|=P_{\lambda}\left[M\left(1+\lambda \varepsilon|c|+\lambda \varepsilon^{2}|c|^{2}\right)\right]
$$

where $\lambda$ stands for $\lambda=\lambda(\varepsilon|c|)$ of Theorem 1. Since $P_{\lambda}$ is nondecreasing, this gives for every $x$ such that $x \cdot c \geqq-R|c|$

$$
u_{\lambda, c}\left(x, t_{0}\right) \geqq P_{\lambda}^{-1}\left(|c|^{2} t-R|c|\right)=M \cdot\left(1+\lambda \varepsilon|c|+\lambda \varepsilon^{2}|c|^{2}\right),
$$

whence

$$
f_{\varepsilon}\left(x, t_{0}\right)=\frac{u_{\lambda, c}\left(x, t_{0}\right)}{1+\lambda \varepsilon c \cdot v+\lambda \varepsilon^{2}|c|^{2}} \geqq f_{0}(x, v), \quad v \in S^{N-1} .
$$

Since $f_{0}$ vanishes identically for $x \cdot c \leqq-R|c|$ and all $v \in S^{N-1},(2.5)$ is true on $\mathbf{R}^{N} \times S^{N-1}$. Of course, for a given time $t>0, f_{\varepsilon}(\cdot, v, t)$ vanishes for all large $|x|$ and all $v \in S^{N-1}$, since the speed of propagation for $(0.1)$ is at most $1 / \varepsilon$. We now use the comparison principle together with Theorem 1 to conclude that for all $t>0$ and $(x, v) \in \mathbf{R}^{N} \times S^{N-1}$,

$$
f_{\epsilon}(x, v, t) \leqq \frac{u_{\lambda, c}\left(x, t_{0}+t\right)}{1+\lambda \varepsilon c \cdot v+\lambda \varepsilon^{2}|c|^{2}} .
$$

Since (2.7) holds for any vector $c$ of fixed norm satisfying (2.4), we conclude from (2.7) that $\operatorname{supp} f_{\varepsilon}(\cdot, v, t) \subset B_{\tilde{R}(t, c)}$, where $\tilde{R}(t)=|c|\left(t+t_{0}\right), t_{0}$ given by (2.5). Therefore, we get an estimate for $R(t)$ of the form

$$
R(t) \leqq R+|c| t+\frac{1}{|c|} P_{\lambda}\left(M\left(1+\lambda \varepsilon|c|+\lambda \varepsilon^{2}|c|^{2}\right)\right) .
$$

To obtain estimate (2.2), we have to choose suitable velocities $c$ in formula (2.8). Thus, when $t=t_{1}$ is large, more precisely, when $\delta \equiv P_{N}(M) \varepsilon^{2} / t_{1}<\delta_{0}=$ $\left(\min \left(1,|N-2|^{-1}\right)\right)^{2}$,

$$
|c|=\left(\frac{P_{N}(M)}{t_{1}}\right)^{1 / 2}
$$

satisfies (2.4) since $\varepsilon|c| \leqq \delta^{1 / 2}$. Moreover, if $\delta$ is small, since $\varepsilon|c|=\delta^{1 / 2} \approx 0, \lambda(\varepsilon|c|)$ is close to $N$ (see Remark 1, Sect. 1), so that (2.8) gives for $t=t_{1}$,

$$
\begin{aligned}
R(t) & \leqq R+\left(P_{N}(M) t\right)^{1 / 2}+\frac{P_{\lambda}\left(M\left(1+0\left(P_{N}(M) \varepsilon^{2} / t\right)^{1 / 2}\right)\right)}{\left(P_{N}(M)\right)^{1 / 2}} t^{1 / 2} \\
& \leqq R+\left(P_{N}(M) t\right)^{1 / 2}+\frac{N}{\lambda} P_{N}\left(M+c_{N} M \delta^{12}\right)^{1 / 2} t^{1 / 2}
\end{aligned}
$$

On the other hand, if $P \varepsilon^{2} / t_{1} \geqq \delta_{0}$, we use the fact that the propagation speed is at most $1 / \varepsilon$ to get

$$
R\left(t_{1}\right) \leqq R+\frac{1}{\varepsilon} t_{1}=R+\left(\frac{P_{N}(M)}{\delta_{0}}\right)^{1 / 2} t^{1 / 2} .
$$

This completes the proof.\# 


\section{Expansion of the Support}

We devote this section to prove that the support of the solutions expands in the variables $x, v$ as $t$ grows. In fact, the support expands instantaneously in the $v$ variable to the whole sphere $S^{N-1}$ at all points where the average $\tilde{f}(x, 0)$ is positive. With respect to the $x$ variable, we show that is does not shrink by means of comparison with suitable subsolutions that we construct. In fact, the solution expands and as $t \rightarrow \infty$ its support becomes the whole space $\mathbf{R}^{n}$. We obtain power-type bounds for the expansion of the support and the decay of the solution.

We begin by establishing the instantaneous expansion in $v$.

Theorem 4. Let $f$ be a nonnegative of $(0.1)_{\varepsilon}, 0<\varepsilon<1$, such that

$$
f(x, v, 0) \geqq \varphi(v), \quad \tilde{\varphi} \geqq \alpha>0
$$

for $x \in B_{r}=B_{r}\left(x_{0}\right), x_{0} \in \mathbf{R}^{N}, r>0$ and $v \in S^{N-1}$. Then

$$
f\left(x, v, \varepsilon^{2} t_{0}\right) \geqq \frac{\alpha}{2}\left(1-e^{-\sigma(\alpha / 2) t_{0}}\right)>0
$$

for every $x \in B_{r / 2}, v \in S^{N-1}$ and $0<t_{0}<r / 2 \varepsilon$.

Proof of Theorem 4. We introduce the solution $g(x, v, t)$ of the linear problem

$$
\left\{\begin{array}{l}
\partial_{t} g+\varepsilon v \cdot \nabla g+\sigma(\alpha / 2) g=\frac{\alpha}{2} \sigma(\alpha / 2), \\
g(x, v, 0)=f(x, v, 0) .
\end{array}\right.
$$

We are going to prove (i) that for any $t_{0}>0, g\left(x, v, t_{0}\right)$ is positive in $B_{r / 2} \times S^{N-1}$, and (ii) that $g\left(x, v, \varepsilon^{2} t\right)$ is a subsolution to $(0.1)_{\varepsilon}$. We conclude the proof using the comparison principle

(i) Observe first that for $t_{0}>0$, we obtain

$$
g\left(x, v, t_{0}\right) \geqq \frac{\alpha}{2} \sigma\left(\frac{\alpha}{2}\right) \int_{0}^{t_{0}} e^{\sigma(\alpha / 2)\left(s-t_{0}\right)} d s=\beta\left(t_{0}\right)=\frac{\alpha}{2}\left(1-e^{-\sigma(\alpha / 2) t_{0}}\right)
$$

by integrating (3.3) along characteristics and recalling that $f(x, v, 0) \geqq 0$.

(ii) In the same way, we get

$$
g(x, v, t) \geqq f(x-\varepsilon v t, v, 0) e^{-\sigma(\alpha / 2) t}+\frac{\alpha}{2} \sigma\left(\frac{\alpha}{2}\right) \int_{0}^{t} e^{\sigma(\alpha / 2)(s-t)} d s .
$$

Thus, for $\left|x-x_{0}\right|+\varepsilon t \leqq r$,

$$
\tilde{g}(x, t) \geqq e^{-\sigma(\alpha / 2) t} \alpha+\frac{\alpha}{2}\left(1-e^{-\sigma(\alpha / 2) t}\right) \geqq \frac{\alpha}{2} .
$$

It follows from (3.3), (3.5) and (H3) that

$$
\varepsilon^{2} \partial_{t} g+\varepsilon v \cdot \nabla g+\sigma(\tilde{g}) g \leqq \sigma(\tilde{g}) \tilde{g},
$$

if $\tau=\varepsilon^{2} t$ and $\left|x-x_{0}\right|+\varepsilon t \leqq r$. Hence $g\left(x, v, \tau / \varepsilon^{2}\right)$ is a subsolution to $(0.1)_{\varepsilon}$ in $\left|x-x_{0}\right| \leqq r-\tau / \varepsilon$. 
(iii) We recall now that the speed of propagation for Eq. $(0.1)_{\varepsilon}$ is bounded above by $1 / \varepsilon$ and use a simple variation of the comparison principle to conclude that

$$
f_{\varepsilon}\left(x, v, \varepsilon^{2} t\right) \geqq g(x, v, t)
$$

for every $x \in B_{r}\left(x_{0}\right)$ and $t>0$ such that $\left|x-x_{0}\right|+\varepsilon t \leqq r$. This applies in particular if $0<t \leqq r / 2 \varepsilon$ and $\left|x-x_{0}\right|<r / 2$.\#

We now want to show that once the solution becomes strictly positive in a certain ball, uniformly in $v$, it will continue to be so for all times. More precisely, our result is

Theorem 5. Let $f$ be a nonnegative function such that

$$
f(x, v, t) \geqq c \text { for } x \in B_{R_{0}}, \quad v \in S^{N-1} .
$$

Then for every $t>0, f(., ., t)$ is strictly positive in $B_{R(t)} \times S^{N-1}$, where $R(t)$ is an increasing function such that

$$
\liminf _{t \rightarrow \infty} \frac{\log R(t)}{t} \geqq \frac{1}{N+2} .
$$

On the other hand, $f$ decays no faster than $P^{-1}\left(t^{-N /(N+2)}\right)$.

Remark. With better information on $\sigma$, we can obtain finer growth rates for $R$. Thus, if we know that for some $s \leqq 1$

$$
s P_{N}(u) \geqq \frac{1}{N \sigma(u)},
$$

then we get instead of (3.7),

$$
\liminf _{t \rightarrow \infty} \frac{\log R(t)}{t} \geqq \frac{1}{N s+2}
$$

We observe that (H3) implies that (3.8) holds for $s=1$. Observe also that as $s \rightarrow 0$, we get the $t^{1 / 2}$-rate that is best possible since it was obtained in Sect. 2 as an upper rate.

Proof of Theorem 5. We will construct a subsolution $h$ to $(0.1)_{\varepsilon}$ which has support in a small ball $B_{R_{0}}$ as $t=0$ and is strictly positive in balls of radius $R(t)$ for time $t>0$ with $R(t)$ increasing and $R(t) \rightarrow+\infty$ as $t \rightarrow+\infty$. $h$ will satisfy the inequality $h_{t}+(1 / \varepsilon) v \cdot \nabla h+\left(1 / \varepsilon^{2}\right) \sigma(\tilde{h})(h-\tilde{h}) \leqq 0$ in $S=\{(x, t): 0<t<\infty,|x|<R(t)\}, h=0$ for $|x|=R(t)$ and $h(x, 0) \leqq f(x, 0)$. Since $f \geqq 0$, we conclude by comparison that $f(x, t, v) \geqq h(x, t, v)$ everywhere. We take $h$ of the form

$$
h(x, t)=U(x, t) g(x, v, t)
$$

with

$$
U(x, t)=P_{N}^{-1}(p(x, t))
$$

where $P_{N}$ is defined in (1.2), and

$$
g(x, v, t)=\frac{\lambda(x, t)}{1+N \varepsilon v \cdot \nabla p}
$$


with $\lambda$ determined so as to have $f g(x, v, t) d v=1$ for every $x, t$. Moreover $p$ will have the typical form of porous media pressures

$$
p(x, t)=\frac{\left(A(t+T)^{2 a}-B|x|^{2}\right)_{+}}{(T+t)} .
$$

Observe that this means that

$$
\begin{gathered}
R^{2}(t)=\frac{A}{B}(t+T)^{2 a}, \quad R_{0}=\frac{A}{B} T^{2 a} \\
\|p(\cdot, t)\|_{\infty}=A(t+T)^{2 a-1} \\
\|\nabla p(\cdot, t)\|_{\infty}=\frac{2 B R(t)}{(T+t)}=2 \sqrt{A B}(T+t)^{a-1} .
\end{gathered}
$$

We prove that $p$ is a suitable subsolution for appropriate values of $A, B, a, T$, which concludes the proof of the theorem

Lemma 6. There exist values of $A, T, B$ and $a$ such that $h$ given by (3.10)-(3.12) is a subsolution of $(0.1)_{\varepsilon}, \varepsilon>0$. Moreover, both $R(0)$ and $\|p(\cdot, 0)\|_{\infty}$ can be made as small as desired. In particular, we may take a arbitrarily close to $1 /(N+2)$ and $B$ arbitrarily close to $1 / 2(N+2)$. The estimates are uniform as $\varepsilon \rightarrow 0$.

Proof of Lemma 6. We want to prove that $h$ satisfies the inequality

$$
\partial_{t} h+\frac{1}{\varepsilon} v \cdot \nabla h+\frac{1}{\varepsilon^{2}} \sigma(\tilde{h})(h-\tilde{h}) \leqq 0 .
$$

1st step. Since $h=U \cdot g$ and $\tilde{h}=U,(3.16)$ can be written as

$$
U_{t}+\frac{1}{\varepsilon} v \cdot \nabla U+\frac{1}{\varepsilon^{2}} \sigma(U) U+\frac{U}{g}\left[g_{t}+\frac{1}{\varepsilon} v \cdot \nabla g\right) \leqq \frac{\sigma(U) U}{\varepsilon^{2} g}
$$

Now $p_{t}=U_{t} /(N \sigma(U))$ and $\nabla p=\nabla U /(N u \sigma(U))$, hence

$$
p_{t}+\frac{1}{\varepsilon} v \cdot \nabla p+\frac{1}{N \varepsilon^{2}} \leqq \frac{1}{N \varepsilon^{2} g}+I,
$$

where

$$
I=-\frac{1}{N \sigma(U)}\left[\frac{g_{t}}{g}+\frac{1 v \cdot \nabla g}{\varepsilon}\right]
$$

We have $1 / g=(1+N \varepsilon v \cdot \nabla p) \lambda^{-1}$, with

$$
\frac{1}{\lambda}=f \frac{d v}{1+N \varepsilon v \cdot \nabla p}=f \frac{d v}{1-N^{2} \varepsilon^{2} v_{1}^{2}|\nabla p|^{2}}=\phi\left(\varepsilon^{2} N^{2}|\nabla p|^{2}\right) .
$$

It is easy to see that $\phi(s)$ is a smooth function of $s$ for $0 \leqq s<1$ with $\phi(0)=1$, $\phi^{\prime}(0)=1 / N$ and $\phi^{\prime \prime}(s) \geqq 0$. Therefore

$$
\frac{1}{\lambda} \geqq 1+\varepsilon^{2} N|\nabla p|^{2}
$$


Substituting into (3.17), we see that the inequality is satisfied if we have

$$
p_{t} \leqq|\nabla p|^{2}+\varepsilon N(v \cdot \nabla p)|\nabla p|^{2}+I .
$$

We turn our attention to $I$. We have

$$
\begin{aligned}
I & =-\frac{1}{N \sigma(U)}\left[\frac{\lambda t}{\lambda}+\frac{1}{\varepsilon} \frac{v \cdot \nabla \lambda}{\lambda}-\frac{N \varepsilon}{z} v \cdot \nabla p_{t}-\frac{N}{z} v \cdot \nabla(v \cdot \nabla p)\right] \\
& =\frac{N \lambda \varepsilon^{2} \phi^{\prime}(s)}{\sigma(U)}\left[2 \nabla p \cdot \nabla p_{t}+\frac{1}{\varepsilon} v \cdot \nabla\left(|\nabla p|^{2}\right)\right]+\frac{\varepsilon}{\sigma(U) z} v \cdot \nabla p_{t}+\frac{1}{\sigma(U) z} v \cdot \nabla(v \cdot \nabla p),
\end{aligned}
$$

where $s=\varepsilon^{2} N^{2}|\nabla p|^{2}$ and $z=1+N \varepsilon v \cdot \nabla p$. We now write (3.21) in the form

$$
\begin{aligned}
p_{t} \leqq & \frac{1}{\sigma(U) z} v \cdot \nabla(v \cdot \nabla p)+|\nabla p|^{2}+\varepsilon N(v \cdot \nabla p)|\nabla p|^{2}+\varepsilon \frac{N \lambda \phi^{\prime}(s)}{\sigma(U)} v \cdot \nabla\left(|\nabla p|^{2}\right) \\
& +\frac{\varepsilon}{\sigma(U) z} v \cdot \nabla p_{t}+\varepsilon^{2} \frac{2 N \lambda \phi^{\prime}(s)}{\sigma(U)} \nabla p \cdot \nabla p_{t} .
\end{aligned}
$$

It is easy to see at this stage the motivation of our choice of subsolution. In fact, with $p$ given by (3.12), it happens that

$$
v \cdot \nabla(v \cdot \nabla p)=\frac{1}{N} \Delta p=-\frac{2 B(t)}{t+T} .
$$

If we disregard the terms in $\varepsilon$ and $\varepsilon^{2}$ as $\varepsilon \rightarrow 0$, we formally obtain

$$
P_{t} \leqq \frac{1}{N \sigma(U)} \Delta p+|\nabla p|^{2}
$$

which means in terms of $U$ that $U_{t} \leqq \Delta F(U)$, i.e. $U$ should be a subsolution of the corresponding porous medium equation.

2nd step. We now justify that, for suitable $A, B, T$ and $a, p$ in (3.12) satisfies (3.22) in $S$ for $\varepsilon>0$. We take $\alpha>0$ small and determine the values of $a$ and $B$ by

$$
\left\{\begin{aligned}
a & =\frac{1-\alpha}{N(1+\alpha)+2(1-\alpha)}<\frac{1}{N+2} \\
2 B & =\frac{1}{N(1+\alpha)+2(1-\alpha)} .
\end{aligned}\right.
$$

Both $a$ and $B$ are positive. By choosing appropriately $A$ (small) and $T$ (large), we will require that $R_{0}=(A / B)^{1 / 2} T^{a}$ be small enough. We also impose that

$$
|\nabla p|=\frac{2 B|x|}{T+t} \leqq \frac{2 B R_{0}}{(T+t)^{1-a} T^{a}} \leqq \frac{2 B R_{0}}{T} \leqq \frac{\delta}{N},
$$

for $\delta$ small enough depending on $\alpha$; this is achieved by choosing $T$ large. We obtain, noticing that $-\Delta p=2 N B /(T+t)$ is positive, $\varepsilon \leqq 1,1 / z \leqq 1+2 \delta, \phi^{\prime}(s) \leqq 2 / N$ 
for $\delta$ small enough, $\lambda \leqq 1$,

$$
\begin{gathered}
-\frac{1}{\sigma(U)} v \cdot \nabla(v \cdot \nabla p)=\frac{-\Delta p}{N \sigma(U) z} \leqq \frac{-\Delta p}{\sigma(U)}(1+2 \delta), \\
\left.\left.|\varepsilon N(v \cdot \nabla p)| \nabla p\right|^{2}|\leqq \delta| \nabla p\right|^{2}, \\
\frac{\left|\varepsilon N \lambda \phi^{\prime}(s) v \cdot \nabla\left(|\nabla p|^{2}\right)\right|}{\sigma(U)} \leqq \frac{2 \lambda}{\sigma(U)} \cdot \frac{8 B^{2}|x|}{(T+t)^{2}} \leqq \frac{4|\Delta p|}{\sigma(U) N}|\nabla p| \leqq \frac{4 \delta|\Delta p|}{\sigma(U) N^{2}} \\
\left|\frac{\varepsilon}{\sigma(U) z} v \cdot \nabla p_{t}\right| \leqq \frac{2 \varepsilon B|x|}{\sigma(U) z(t+T)^{2}}=\frac{2(1+2 \delta)}{B \sigma(U) N}|\nabla p||\Delta p| \leqq \frac{3 \delta}{B \sigma(U) N^{2}}|\Delta p| \\
\left|\frac{\varepsilon^{2} 2 N \lambda \phi^{\prime}(s) \nabla p \cdot \nabla p}{\sigma(U)}\right| \leqq \frac{4}{\sigma(U)}|\nabla p| \frac{2 B|x|}{(t+T)^{2}} \leqq \frac{2 \delta^{2}|\Delta p|}{\sigma(U) B N^{3}} .
\end{gathered}
$$

Finally, we use the estimate $p \geqq 1 / N \sigma(U)$, and (3.22) is satisfied if

$$
p_{t}-\left(1-\varepsilon \delta|\nabla p|^{2}\right)-\frac{4 p \Delta p}{N \sigma(U)}(1+C \delta) \leqq 0
$$

for some universal constant $C$. For small $\delta$ and large $T$, it is enough to check that

$$
P_{t}-(1-\alpha)|\nabla p|^{2}-(1+\alpha) p \Delta p \leqq 0 .
$$

Finally, it is easy to check that, with our previous choice of $B$ and $a, p$ given by (3.12) is an exact solution of (3.25); on the other hand, the parameters $A$ and $T$ are only required to be small and large respectively, in order that $R_{0}$ and $\|p(\cdot, 0)\|_{\infty}$ be small enough.\#

Remark. A careful inspection of our proof shows that the support increases strictly even near $t=0$ when the initial "pressure" $P_{N}\left(f_{0}\right)$ vanishes at the boundary in a superlinear way, but it is not excluded that waiting times occur when for instance $P_{N}\left(f_{0}\right)=O\left(d^{2}\right)$, where $d$ is the distance to the boundary (see [12] for an analogous situation in the P.M.E.).

\section{Appendix. Comparison Principle for the Radiative Transfer Equations}

In this appendix, we recall the proof of the comparison principle for solutions to the Radiative Transfer Equations. We refer to Mercier [9] or to [6] for the original proof.

Let $f(x, v, t), g(x, v, t)$ satisfy

$$
\begin{gathered}
0 \leqq f, g ; \quad f, g \in L^{1}\left(\mathbf{R}^{N} \times S^{N-1} \times[0, T]\right), \\
\partial_{t} f+v \cdot \nabla f+\sigma(\tilde{f})(f-\tilde{f})=S(x, v, t), \\
\partial_{t} g+v \cdot \nabla g+\sigma(\tilde{g})(g-\tilde{g}) \leqq S(x, v, t), \\
0 \leqq g(x, v, 0) \leqq f(x, v, 0) \in L^{1}\left(\mathbf{R}^{N} \times S^{N-1}\right),
\end{gathered}
$$


and we assume that (A.2), (A.3) hold in $L^{1}$, i.e.

$$
\begin{gathered}
\sigma(\tilde{f}) \tilde{f}, \sigma(\tilde{g}) \tilde{g} \in L^{1}\left(\mathbf{R}^{N} \times[0, T]\right), \\
0 \leqq S, \quad S \in L^{1}\left(\mathbf{R}^{N} \times S^{N-1} \times[0, T]\right) .
\end{gathered}
$$

We claim that if $\sigma$ satisfies (H1), (H3), then

$$
g(x, v, t) \leqq f(x, v, t) \quad \text { a.e. }
$$

Proof of (A.6). Following [9], we introduce

$$
\Gamma=\operatorname{sgn}^{-}(f-g),
$$

where

$$
t^{-}=\sup (0,-t), \operatorname{sgn}^{-} t=-1_{t^{-}>0} .
$$

then, from (A.2), (A.3) and since $\partial_{t}(f-g)+v \cdot \nabla(f-g)$ lies in $L^{1}$, we get multiplying by $\Gamma$,

$$
\left(\partial_{t}+v \cdot \nabla\right)(f-g)^{-}+\Gamma[\sigma(\tilde{f}) f-q(\tilde{f})-\sigma(\tilde{g}) g-q(\tilde{g})] \leqq 0,
$$

where

Setting

$$
q(t)=t \sigma(t) \text { is non-decreasing. }
$$

$$
\bar{\Gamma}=\operatorname{sgn}^{-}(\tilde{f}-\tilde{g}) ; \quad(\bar{\Gamma}=\Gamma(x, t))
$$

and integrating (A.7) in $x$ and $v$, we get

$$
\partial_{t} \int_{x, v}(f-g)^{-} d x \frac{d v}{\left|S^{N-1}\right|}+\int_{x} f \Gamma[\sigma(\tilde{f}) f-q(\tilde{f})-\sigma(\tilde{g}) g-q(\tilde{g})] d x d v \leqq 0 .
$$

But the integral in $v$ in the second term of this inequality may be written as

$$
\begin{aligned}
& f \Gamma[\sigma(\tilde{f}) f-q(\tilde{f})-\sigma(\tilde{g}) g-q(\tilde{g})]+f \bar{\Gamma}(x, t)[q(\tilde{f})-\sigma(\tilde{f}) f-q(\tilde{g})+\sigma(\tilde{g}) g] \\
&= f\left\{[q(\tilde{f})-q(\tilde{g})]^{-}-\Gamma[q(\tilde{f})-q(\tilde{g})]\right\} d v \\
&+f \sigma(\tilde{f})\left[(f-g)^{-}-\bar{\Gamma}(f-g)\right] d v \\
&+f g\left\{-\Gamma(\sigma(\tilde{g})-\sigma(\tilde{f}))+[\sigma(\tilde{g})-\sigma(\tilde{f})]^{-}\right\} d v
\end{aligned}
$$

(since $\sigma$ is non-increasing). Now, every of these three integrals is non-negative (since the terms inside are non-negative). This shows that

$$
\partial_{t} \int_{x, v}(f-g)^{-} d x d v \leqq 0,
$$

and thus that $(f-g)^{-} \leqq 0$, i.e. $f \geqq g$.

Since the R.T.E. has a finite speed of propagation, it is classical to deduce, using similar arguments, a local version of (A.6). Namely, if (A.2), (A.3) are satisfied in a cylinder $C=B_{r} \times S^{N-1} \times[0, T]$, then the conclusion (A.6) holds on the set $E$

$$
E=\left\{(x, v, t) ;|x| \leqq r-t, v \in S^{N-1}, t \in[0, T]\right\} .
$$


This is used in a crucial way to prove Lemma 5. Also, if (A.2), (A.3) are satisfied in $C$, and

$$
g(x, v, t) \leqq f(x, v, t), \quad \forall t \in[0, T], \quad v \in S^{N-1}, x \in \partial B_{r} \quad \text { with } \quad x \cdot v \leqq 0,
$$

then (A.6) holds in C. This is used in Lemma 6.

Acknowledgement. This work was initiated while the first author was visiting the Universidad Autonoma de Madrid. He wishes to thank that university and Universite Paris IX-Dauphine for making the visit possible.

\section{References}

1. Aronson, D. G.: The porous medium equation. In: Nonlinear Diffusion Problems. Lecture Notes in Mathematics, vol. 1224 C.I.M.E. Series, Berlin, Heidelberg, New York: Springer 1986

2. Bardos, C., Golse, F., Perthame, B.: The Rosseland approximation for the radiative transfer equations. Commun. Pure Appl. Math. 40, 691-721 (1987)

3. Bardos, C., Golse, F., Perthame, B., Sentis, R.: The nonaccretive radiative transfer equations. Existence of solutions and Rosseland approximation. Funct. Anal. 77, 434-460 (1988)

4. Diaz, J. I.: Solutions with compact support for some degenerate parabolic problems. Nonlin. Anal. 3, 831-847 (1979)

5. Gilding, B., Peletier, L. A.: On a class of similarity solutions of the porous medium equation. J. Math. Ann. Appl. 55, 351-364 (1976)

6. Golse, F., Perthame, B.: Generalized solutions of the radiative transfer equations. Commun. Math. Phys. 106, 211-239 (1986)

7. Kalashnikov, A.S.: On equations of the nonstationary filtration type in which the perturbations are propagated at infinite velocity. Vestnik Mosk. Univ. 27, 104-108 (1972) (in Russian)

8. Knerr, B. F.: The porous medium equation in one dimension. Trans. Am. Math. Soc. 234, 381-415 (1977)

9. Mercier, B.: Applications of accretive operator theory to the radiative transfer equations. S.I.AM. J. Math. Anal., 18, 393-408 (1987)

10. Oleinik, O. A., Kalashnikov, A. S., Czhou, Y. L.: The Cauchy problem and boundary value problems for equations of the type of unsteady filtration. Izv. Akad. Nauk. SSSR, Ser. Mat. 22, 667-704(1958)

11. Peletier. J. L.: A necessary and sufficient condition for the existence of an interface in flows through porous media. Arch. Rat. Mech. Anal. 56, 183-190 (1974)

12. Vazquez, J. L.: The interface of one-dimensional flows in porous media. Trans. Am. Math. Soc. 285, 717-737 (1984)

Communicated by J. L. Lebowitz

Received August 1, 1989 
